\title{
ESTUDIO DE CASOS DE LA ENSEÑANZA DE LA LENGUA Y CULTURA MAPUCHE Y SU IMPLEMENTACIÓN COMO ASIGNATURA DEL CURRÍCULO DE EDUCACIÓN BÁSICA ${ }^{1}$
}

\author{
Margarita Calderón ${ }^{2}$ \\ Silvia Castillo ${ }^{3}$ \\ Diego Fuenzalida ${ }^{4}$ \\ Felipe Hasler ${ }^{5}$ \\ Héctor Mariano ${ }^{6}$ \\ Cristián $\operatorname{Vargas}^{7}$
}

RESUMEN

Esta investigación indaga en los contenidos y estrategias de enseñanza de la cultura y la lengua mapuche a través de las percepciones y prácticas de las duplas pedagógicas que implementan la asignatura de Lengua Indígena en dos niveles del primer ciclo básico. Para ello, se seleccionaron seis escuelas con implementación del sector curricular, que cumplieran con los siguientes criterios de selección: 1) implementación efectiva, 2) zona geográfica, 3) antigüedad de implementación del sector y/o iniciativas sobre interculturalidad y bilingüismo. En cada establecimiento se realizaron entrevistas a directivos, educadores tradicionales y docentes, además de observación de clases. Respecto de las estrategias de enseñanza, se identificaron dos tipos: las estrategias vinculadas con la cultura mapuche y aquellas no vinculadas directamente con esta cultura. Los contenidos de enseñanza en las distintas escuelas son muy similares, aunque contextualizados en función de la realidad local; no obstante, enfatizan principalmente en los contenidos culturales mapuche más que en elementos propios de la enseñanza de la lengua originaria. Por la autonomía con la que trabajan, los educadores generan

1 Se agradece el financiamiento otorgado por el Fondo Basal para Centros de Excelencia proyecto FB0003 de PIA-CONICYT, Programa de Cooperación Bilateral Chile - Federación Valonia-Bruselas y región Valona de Bélgica y del Consejo Nacional de Educación (CNED) a través de su Convocatoria de apoyo a la investigación en educación 2016.

2 Departamento de Estudios Pedagógicos - Centro de Investigación Avanzada en Educación, Universidad de Chile, Santiago, Chile. Contacto: margarita.calderon@ciae.uchile.cl

3 Facultad de Educación, Universidad Católica Silva Henríquez, Santiago, Chile. Contacto: scastillos@ucsh.cl

4 Centro de Investigación Avanzada en Educación, Universidad de Chile, Santiago, Chile. Contacto: dfuenzalida@ciae.uchile.cl

5 Centro de Investigación Avanzada en Educación, Universidad de Chile, Santiago, Chile. Contacto: fhasler@uchile.cl

6 Centro de Investigación Avanzada en Educación, Universidad de Chile, Santiago, Chile. Contacto: hmariano@uchile.cl

Centro de Investigación Avanzada en Educación, Universidad de Chile, Santiago, Chile. Contacto: cristianvp07@gmail.com 
una reflexión propia para tomar decisiones de carácter pedagógico que está orientada a motivar en sus estudiantes el sentido de pertenencia al pueblo mapuche antes que en la generación de bilingüismo.

Palabras clave: asignatura lengua indígena, contenidos de enseñanza, estrategias de enseñanza, estudio de casos, mapuzugun.

\title{
TEACHING THE LANGUAGE AND CULTURE OF THE MAPUCHE: A CASE STUDY OF ITS IMPLEMENTATION AS A SUBJECT IN THE PRIMARY EDUCATION CURRICULUM
}

\begin{abstract}
This research explores the content and teaching strategies of the language and culture of the Mapuche, taught in primary school, by analyzing the perceptions and practices of the pedagogical pairs who implement the subject of Indigenous Language in the early years of primary education. The sample was comprised of six schools who offer this subject, that met the following selection criteria: 1) effective implementation, 2) geographic zone; 3) number of years implementing the subject and/or initiatives on intercultural education and bilingualism. In each school, classroom observations and interviews with the school leaders, traditional educators and teachers were conducted. The research results showed there are two main types of teaching strategies: those related to the mapuche culture and those which are not directly related to this culture. The teaching contents are very similar among all the cases; however, they are contextualized to the local contexts of each community. In addition, the contents are predominantly oriented towards cultural, instead of language. Due to the autonomy in which the traditional educators work, their pedagogical decisions are based on their own beliefs on how to motivate students to develop a sense of belonging towards the mapuche identity instead of focusing on bilingualism.
\end{abstract}

Keywords: Indigenous language subject, instructional content, teaching strategies, case study, mapuzugun

\section{Introducción}

Han transcurrido siete años desde el comienzo de la implementación de la asignatura de Lengua Indígena mapuzugun ${ }^{8}$ en Chile. Los primeros diagnósticos identificaron que existe escasa información respecto de los contenidos de enseñanza en la asignatura y cómo estos son transmitidos, considerando que existe una diversidad de realidades locales (Centro de Políticas Comparadas de

8 En este artículo las palabras en lengua mapuzugun se escribirán con cursiva y utilizando el grafemario azümchefe, que es utilizado por el Estado chileno para escribir documentos oficiales en dicha lengua. 
Educación, CPCE, 2012; Dirección de Presupuestos, Dipres, 2013; Sotomayor, Allende, Castillo, Fuenzalida y Hasler, 2013). Dados estos antecedentes, la presente investigación tiene como objetivos identificar contenidos y estrategias de enseñanza de la cultura y la lengua mapuche a través de percepciones y prácticas de las duplas pedagógicas de seis escuelas que implementan la asignatura de Lengua Indígena y analizar el uso de recursos pedagógicos provistos por el Ministerio de Educación de Chile (Mineduc) en la implementación de la asignatura de lengua mapuche.

El artículo se estructura de la siguiente manera: antecedentes; marco de referencia; metodología; resultados; discusión y conclusiones.

\section{Antecedentes}

\subsection{Políticas educativas para los pueblos originarios en Chile}

En un escenario global en el que tanto los organismos internacionales como los movimientos indígenas reivindican los derechos de los pueblos originarios, se promulga en Chile la Ley Indígena $N^{\circ} 19.253$ en 1993, que sienta las bases para la instauración de la Educación Intercultural Bilingüe (EIB) en el país (Organización de las Naciones Unidas, ONU, 1948; 1966, 1989, 2007; Organización de las Naciones Unidades para la Educación, Ciencia y Cultura, Unesco, 2000, 2005; Organización Internacional del Trabajo, OIT, 1989). Desde este hito se desarrolla en el Mineduc, entre 1996 y 1999, una unidad que implementa un plan piloto, a partir del cual posteriormente se consolida el Programa de Educación Intercultural Bilingüe (PEIB). Con la puesta en marcha del Programa Multisectorial Orígenes, en el año 2001, se le asignan fondos a esta unidad y pasa a denominarse PEIB-Orígenes.

En una siguiente etapa, a fines del año 2009, el Mineduc aprueba el Decreto 280 que crea la asignatura de Lengua Indígena, que implica la enseñanza del mapuzugun, quechua, aymara y rapa nui, con un carácter obligatorio en todos los establecimientos con más de 
un $20 \%$ de estudiantes de ascendencia indígena y opcional para todas las escuelas que deseen implementarlo. En el año 2010 comenzó con $1^{\circ}$ año básico y debía aumentar progresivamente de nivel hasta llegar a $8^{\circ}$ año básico en 2017 . Una característica relevante de la asignatura es que debe ser implementada por una dupla pedagógica compuesta por un/a educador/a tradicional (ET), que enseña la lengua, y un profesor mentor (PM), docente de aula, que aporta las competencias pedagógicas. Finalmente, este decreto plantea tres posibles estrategias de focalización pertinentes para el pueblo mapuche: a) Asignatura de Lengua Indígena, orientada a escuelas rurales en zonas de alta concentración indígena; b) Interculturalidad, línea que busca rescatar la lengua indígena y favorecer la interculturalidad, principalmente en zonas urbanas; y c) Bilingüismo.

\subsection{La implementación de la asignatura de Lengua Mapuzugun}

El mapuzugun es la lengua originaria más hablada en el país (Gundermann, Canihuan, Clavería y Faúndez, 2008; 2009; 2011) y la asignatura de esta lengua es la que mayoritariamente se ha implementado en establecimientos. Esto se relaciona directamente con la mayor presencia mapuche en el sistema escolar: de los nueve pueblos indígenas reconocidos en Chile, el pueblo mapuche concentra el $86,1 \%$ de la matrícula indígena registrada, seguido por un $9 \%$ del pueblo aymara (Carvajal y Lafferte, 2014). Las distintas estrategias de focalización se implementan, principalmente, en las regiones Metropolitana, Biobío, Araucanía, Los Ríos y Los Lagos.

La evidencia acerca de la implementación de la asignatura de Lengua Indígena Mapuzugun en Chile y de las otras estrategias de focalización es limitada, pero creciente. Recientes estudios indican que los recursos educativos asociados al sector curricular son considerados como insuficientes o poco pertinentes para abordar las particularidades locales (CPCE, 2012; Sotomayor, Allende, Castillo, Fuenzalida y Hasler, 2014); en general, hay una positiva valoración de la incorporación de la asignatura, no sin ciertas reticencias iniciales de apoderados y profesores. Adicionalmente, algunas de las tareas pendientes dicen relación con la regularización del trabajo 
de los educadores, la participación más activa de las comunidades escolares y mapuche, el aumento de la cobertura de la asignatura, el mejoramiento de las capacitaciones de educadores y profesores, el fortalecimiento de escenarios pedagógicos adecuados para la enseñanza de la lengua y cultura mapuche, la incorporación activa de los directivos en todo el proceso, la falta de normalización de las lenguas indígenas y su baja valoración en comparación con el castellano, entre otros aspectos (CPCE, 2012; Dipres, 2013; Sotomayor et al., 2014; Ibáñez, Rodríguez y Cisternas, 2014).

\section{Marco de referencia}

\subsection{Contenidos y metodologías mapuche en el contexto escolar}

Algunas investigaciones acerca de la asignatura indican que la dupla pedagógica, tanto en zona urbana como rural, integra en sus clases fundamentalmente contenidos culturales mapuche, entre los que destacan el aprendizaje de vocabulario referido a cosmovisión, números, habitación y uso del espacio, estructura familiar y social mapuche, música, danzas, vestimentas y alimentación (Castillo y Loncon, 2015; Cortés y Concha, 2013; Luna, Benavides, Gutiérrez, Alchao y Dittborn, 2014; Quidel, 2011; Sotomayor et al., 2014). Sin embargo, en estos estudios no se detallan los contenidos de enseñanza específicos, así como tampoco la progresión de las materias tratadas para cada nivel, los valores transmitidos ni las habilidades desarrolladas. Por tanto, quedan interrogantes referidas a cómo entender el currículo real (Casarini, 1999), el lugar efectivo que tiene el bilingüismo dentro de la implementación de la EIB (Espinoza, 2016), la relación entre los contenidos de enseñanza y las diversas identidades territoriales y el uso del mapuzugun en el aula.

Por otro lado, se ha observado que, en el contexto de la implementación de la asignatura mapuzugun, el uso de la lengua mapuche se encuentra restringido a la sala de clases en las horas destinadas a la asignatura y a ciertas celebraciones institucionalizadas como el wiñol txipantü (año nuevo mapuche). Lo último, propiciado por las particularidades de la implementación de la asignatura en la 
escuela (Sotomayor et al., 2014). Esta situación se sostiene porque el sistema escolar chileno está pensado desde una lógica monocultural y occidental, excluyendo otros saberes, contenidos y, por ende, la finalidad educativa desde una perspectiva mapuche, de acuerdo con Quilaqueo y Quintriqueo (2010) y Quilaqueo y Torres (2013). Los autores antes citados identifican una distancia epistemológica entre la construcción del conocimiento mapuche y no mapuche, pues el primero se caracteriza por integrar aspectos concretos de la vida real con las fuerzas naturales y sobrenaturales presentes en el universo humano. A la inversa, el conocimiento occidental moderno segmenta en disciplinas cada aspecto del saber. En la misma línea, Quilaqueo (2010) plantea que los contenidos educativos mapuche más destacados son aquellos que inciden en la relación entre los seres humanos y su entorno natural y espiritual. Al mismo tiempo, señala que estos contenidos se transmiten por medio de la memoria oral a través del ngülam (consejo). En síntesis, de acuerdo con estos autores, la enseñanza de los contenidos culturales se basa en una diversidad de estrategias discursivas orales, en un marco holístico de comprensión de los fenómenos, que genera prácticas y normas para comprender conceptos complejos.

\subsection{Estrategias y prácticas de enseñanza}

De acuerdo con Valls (1990, citado en Solé, 1994), la estrategia, al igual que otros procedimientos, permite "seleccionar, evaluar, persistir o abandonar determinadas acciones para llegar a conseguir la meta que nos proponemos" (p. 67). Entre sus particularidades, destacan:

- son independientes a una esfera particular, por tanto, se pueden generalizar;

- involucran autodirección, en otras palabras, la determinación de un objetivo; y

- implican autocontrol, en decir, el monitoreo y evaluación de la acción emprendida, considerando el o los objetivos que la direccionan y la posibilidad de efectuar un cambio en ella, cuando se requiera. 
En el ámbito de la educación, las estrategias se constituyen como globalidades o generalizaciones, amparadas en una teoría o marco conceptual, para dirigir la enseñanza o transmisión de conocimientos. En un nivel más general, las estrategias se enmarcan en un sistema educativo que responde a su vez a valores sociales, culturales y económicos, expresados en las políticas y objetivos del establecimiento educacional, en políticas y programas nacionales, y en las percepciones y participación de los apoderados y de la comunidad en la educación de los niños.

En el estudio de las estrategias de enseñanza intercultural debe tomarse en cuenta la existencia de distintos sistemas de enseñanza, en el marco de sistemas socioculturales diversos. Así también, es importante analizar qué actores participan en la constitución de las estrategias de enseñanza, y de qué manera lo hacen (el sistema occidental y el sistema mapuche).

Las prácticas de enseñanza pueden considerarse como la operacionalización de las estrategias (Lave, 2009), esto es, las actividades y acciones concretas que se llevan a cabo para enseñar a los niños. Implican por lo tanto a quien enseña, al enseñado y al contexto donde se produce la enseñanza. El estudio de las prácticas supone poner atención en el tipo de interacciones que se producen, en los esquemas que se repiten, en las reacciones específicas en cada situación, en el tipo de interacciones que se producen en la enseñanza, entre otros.

Un conjunto de prácticas implica o supone una estrategia. Las estrategias, a su vez, se enmarcan en un campo teórico. El método, entendido como conjunto de operaciones ordenadas con que se pretende obtener un resultado, constituye un conjunto de prácticas vinculadas a una teoría o marco conceptual. En un marco teórico se pueden encontrar varios métodos, los que responden a distintas vertientes en una misma teoría. Por lo tanto, una estrategia, basada en un marco teórico determinado, se puede operacionalizar en varios métodos, donde cada uno comprende un conjunto de prácticas. De acuerdo con Casarini (1999), la propuesta curricular explicitada en los programas de estudio guía la práctica docente y determina lo 
que ocurre en el aula. Sin embargo, el estudio de la implementación curricular involucra diferenciar el currículo oficial del real (Casarini, 1999).

El currículo real representa la realización concreta del currículo formal en el aula. Por otro lado, el currículo oculto (Arciniegas, 1982, citado en Casarini, 1999) corresponde a enseñanzas encubiertas o latentes, que favorecen y propician creencias, valores y posiciones ideológicas de determinados grupos en desmedro de otros, y que a menudo discrimina a partir de la raza, la clase y el género. En el ámbito de la implementación de la asignatura de lengua mapuzugun, aún no existe evidencia respecto de la implementación del currículo formal, es decir, no hay evidencia respecto del currículo real.

En relación con las estrategias utilizadas en la práctica pedagógica, Quidel (2011) describe, analiza y evalúa las estrategias metodológicas de enseñanza del mapuzugun como segunda lengua en el aula, concluyendo que son similares a las de una lengua extranjera (traducción, preguntas y respuestas, lectura y escritura) y que, por tanto, dejarían de lado aquellas estrategias propias de la sociedad mapuche.

Por su parte, Luna y colaboradores (2014) realizan un análisis etnográfico de la implementación de la asignatura de lengua mapuche con foco en la utilización de los recursos educativos en el aula. Respecto de investigaciones referidas a la experiencia en zona urbana, Cortés y Concha (2013) mencionan que los contenidos abordados en los talleres se orientan al aprendizaje de la lengua, pero con un fuerte componente cultural. La oralidad ocupa un lugar privilegiado, expresada en el uso de canciones, narración de cuentos, creación de poemas y relatos.

Por otra parte, Lara (2012) advierte que en las clases de la asignatura Lengua Indígena observadas en La Araucanía, las prácticas pedagógicas en su mayoría solo estaban orientadas a la decodificación de palabras, marginando la posibilidad de abordar la enseñanza de la lengua desde una perspectiva funcional. Un estudio más reciente (Castillo y Loncon, 2015) revela que los ET no solo 
movilizan estrategias no mapuche, sino que hay presencia de un modelo educativo propio, siguiendo la propuesta de Quilaqueo y Quintriqueo (2010). Entre sus prácticas educativas más habituales, el educador, desde su percepción, destaca la utilización de géneros textuales tradicionales y subraya la importancia de la oralidad como un aspecto clave en la enseñanza9.

\section{Metodología}

La metodología considerada para este estudio fue cualitativa tomando el estudio de casos como estrategia central (Robson, 1993). Este enfoque permite informar acerca del contexto y la complejidad de las creencias y prácticas asociadas a la lengua y cultura mapuche en la escuela.

\subsection{Selección de la muestra}

Los estudios de casos se basaron en analizar escuelas que están implementando la asignatura de lengua mapuzugun. La estructura de la muestra es no probabilística y se establece sobre la base de cuatro criterios de selección:

- Implementación efectiva de la asignatura de lengua mapuzugun.

- Evidencia de buenas prácticas.

- Zona geográfica (escuela rural o urbana).

- Antigüedad de implementación de EIB o programas asociados: 1) Escuelas que implementan programas de EIB desde antes de que comenzara la asignatura (etapa PEIB-Orígenes), o escuelas de larga trayectoria en EIB; 2) Escuelas que implementan desde que comenzó la asignatura (año 2010); y 3) Escuelas con implementación reciente de la asignatura (tres años o menos).

\footnotetext{
Vale la pena destacar que, en la presente investigación, las propuestas de modelo educativo propio o de estrategias de enseñanza mapuche no se definen por su correspondencia estricta con modelos educativos mapuche como tales, dado que en la sociedad mapuche no han existido ni existen instituciones tradicionales orientadas a la escolarización, sino más bien se clasifican dentro de esta categoría por ser estrategias de enseñanza ancladas en prácticas culturales propias de las comunidades mapuche, asumiendo que han debido ser adaptadas y refuncionalizadas para ingresar a un nuevo contexto, ajeno y definido desde otras coordenadas epistemológicas, como la escuela. Las tensiones e implicancias de este tipo de adaptaciones, situadas cultural e históricamente, son una proyección de la presente investigación cuyo tratamiento excede los objetivos de esta.
} 
Los dos primeros criterios se consideraron requisitos excluyentes para la selección de las escuelas. De acuerdo con los restantes dos criterios, se seleccionaron los siguientes establecimientos:

Tabla 1

Establecimientos seleccionados para la investigación

\begin{tabular}{|c|c|c|c|c|c|}
\hline Casos & Ubicación & Dependencia & Matrícula & $\begin{array}{c}\text { Zona } \\
\text { geográfica }\end{array}$ & Sigla $^{1}$ \\
\hline $\begin{array}{l}\text { Escuela rural con } \\
\text { implementación } \\
\text { reciente. }\end{array}$ & $\begin{array}{l}\text { San Juan de } \\
\text { la Costa, Los } \\
\text { Lagos. }\end{array}$ & Municipal. & $20^{2}$ & Rural & RR \\
\hline $\begin{array}{l}\text { Escuela rural con } \\
\text { implementación } \\
\text { desde } 2010 .\end{array}$ & $\begin{array}{l}\text { Temuco, La } \\
\text { Araucanía. }\end{array}$ & $\begin{array}{l}\text { Particular } \\
\text { subvencionado. }\end{array}$ & 82 & Rural & RI \\
\hline $\begin{array}{l}\text { Escuela rural con } \\
\text { iniciativas anteriores } \\
\text { a la implementación } \\
\text { de la asignatura. }\end{array}$ & $\begin{array}{l}\text { Padre Las } \\
\text { Casas, La } \\
\text { Araucanía. }\end{array}$ & Municipal. & 59 & Rural & RA \\
\hline $\begin{array}{l}\text { Escuela urbana con } \\
\text { implementación } \\
\text { reciente. }\end{array}$ & $\begin{array}{l}\text { San Ramón, } \\
\text { RM. }\end{array}$ & $\begin{array}{l}\text { Particular } \\
\text { subvencionado. }\end{array}$ & 1.495 & Urbana & UR \\
\hline $\begin{array}{l}\text { Escuela urbana con } \\
\text { implementación } \\
\text { desde el } 2010 .\end{array}$ & $\begin{array}{l}\text { Osorno, Los } \\
\text { Lagos. }\end{array}$ & $\begin{array}{l}\text { Particular } \\
\text { subvencionado. }\end{array}$ & $1.354^{3}$ & Urbana & UI \\
\hline $\begin{array}{l}\text { Escuela urbana con } \\
\text { iniciativas anteriores } \\
\text { a la implementación } \\
\text { de la asignatura. }\end{array}$ & $\begin{array}{l}\text { Padre Las } \\
\text { Casas. La } \\
\text { Araucanía. }\end{array}$ & $\begin{array}{l}\text { Particular } \\
\text { subvencionado. }\end{array}$ & 199 & Urbana & UA \\
\hline
\end{tabular}

Fuente: Elaboración propia.

La recolección de datos en cada establecimiento contempló entrevistas semiestructuradas (Bryman, 2004) a directivos, ET y profesores mentores que enseñen en primero o segundo año básico y en tercero o cuarto año básico, seleccionando dos niveles de acuerdo con lo consensuado con la dupla pedagógica. Se utilizó como

1 Con esta sigla se hará referencia a los establecimientos en el documento. El nombre de los establecimientos ha sido cambiado para resguardar la identidad de los actores entrevistados.

2 Información proporcionada por el director.

3 Información proporcionada por el director. 
principal criterio la variabilidad, tanto de contenidos como estrategias de enseñanza.

Además, se efectuaron al menos cuatro observaciones de clases para los dos niveles seleccionados en cada caso. Las pautas de observación se elaboraron a partir de los objetivos específicos de la investigación y se organizaron en torno a sus temáticas centrales:

- Contenidos mínimos obligatorios y contenidos culturales.

- Estrategias de enseñanza.

- Recursos educativos.

- Evaluación.

La observación de clase se realizó con énfasis en la observación de las estrategias y contenidos de enseñanza y se complementó con notas de campo (Lofland y Lofland, 1995).

Por otra parte, las entrevistas a los directivos tuvieron como propósito comprender el contexto de implementación de la asignatura. Junto con lo anterior, se analizaron las planificaciones anuales de las duplas en conjunto con ellos, en caso de estar disponibles, para triangular la información recolectada en las entrevistas. Los instrumentos se elaboraron a partir de un pilotaje que se realizó durante el año 2016 y comienzos de 2017.

\subsection{Procedimientos de análisis}

El contenido de las entrevistas se analizó por medio de la creación de códigos o categorías asignadas a determinadas piezas de información que son relevantes para los objetivos de la investigación (Friese, 2012). La codificación se realizó con el apoyo del software QSR Nvivo 10.

Esta estrategia de análisis cualitativo se desarrolló a partir del modelo Noticing Thinking Collecting (NTC) (Friese, 2012) y posee dos niveles de análisis: un primer nivel descriptivo y luego un nivel conceptual.

En esta muestra, el análisis descriptivo se realizó utilizando una estrategia top-down, es decir, que se elaboró una lista de temas 
sobre la base de las temáticas centrales de la investigación. Esta lista inicial fue complementada con algunas categorías que surgieron durante el proceso. El segundo nivel de análisis se realizó mediante la creación de relaciones y definiciones que surgieron a partir de las ideas expresadas por los ET, docentes y directivos.

La validación de los datos se realizó en concordancia con la naturaleza del estudio, para ello, se contrastaron los resultados con la literatura especializada y se corroboraron los resultados con los participantes del estudio (Creswell, 2009).

\section{Resultados}

\subsection{Caracterización general de los casos y elementos de contexto}

A continuación, se presenta una breve descripción de cada caso de estudio distinguido por los atributos de trayectoria de iniciativas interculturales y zona geográfica.

4.1.1. Escuela rural de implementación reciente de la asignatura (RR) Se ubica en la comuna de San Juan de la Costa y acoge a 20 niños en un curso multigrado, todos de origen mapuche-williche. Las familias se dedican principalmente a la pesca, recolección de algas o agricultura de subsistencia. Los alumnos asisten desde distintos sectores, algunos desde una gran distancia de la escuela. La planta docente consta de tres profesores y un psicólogo.

La asignatura se implementa en cuatro horas semanales, en donde se enseña mapuzugun, manejo de conceptos y palabras, cosmovisión, efku (ceremonial), awkantun (juegos), entre otros. Según indica el director, estos contenidos se refuerzan en la familia, por lo que, por parte de la escuela, se identifica como una ventaja trabajar con niños provenientes de familias mapuche, ya que hay un fuerte sentido de pertenencia cultural en los estudiantes y sus familias.

En conjunto con la implementación de la asignatura de Lengua Indígena, se realiza un proceso de recontextualización de las demás 
asignaturas de acuerdo con la cultura mapuche. Por ejemplo, en Lenguaje y Comunicación se habla de piam y epew en lugar de "mitos" y "leyendas", y en Música se trabajan contenidos relacionados con el wiñol txipantü entre marzo y junio. El director identifica la necesidad de contextualizar los planes y programas del Mineduc (de la asignatura y en general), de manera que hagan sentido a la realidad de los niños.

El director y la ET indican que el interés en la cultura mapuche y el sentido de pertenencia en la comunidad han ido aumentando gracias a la implementación de la asignatura. Asimismo, la escuela realiza visitas a miembros clave de la comunidad.

4.1.2. Escuela rural que implementa la asignatura desde sus inicios (RI) Se ubica en una comunidad mapuche de la comuna de Temuco y entrega educación básica. Surgida en el año 2000 como un "proyecto educativo multicultural y bilingüe", se integró al PEIB el año 2010, tras generar su propio proyecto curricular intercultural, implementando la asignatura. Según los actores de la escuela, el 98\% de los alumnos es de origen mapuche. La planta docente está constituida por nueve profesores, cinco de ellos mapuche. Se cuenta además con dos asistentes y un equipo de profesionales vinculado al proyecto de integración.

Hay dos ET y su labor contempla la enseñanza del mapuzugun, la participación en el nguillatun, eluwün y machitun y la enseñanza del ngülamtun, esto es, consejos sobre el comportamiento adecuado de los niños, con los adultos mayores y entre pares. Como indica el director, el proyecto educativo de la escuela plantea como misión de la misma revitalizar la cultura mapuche a través de su puesta en valor y mediante la recuperación de la memoria. Para los ET, los objetivos de la asignatura son que los niños se sientan orgullosos de ser mapuche y que defiendan sus derechos como tales. Por otro lado, el director identifica como una de las dificultades de la asignatura las precarias condiciones laborales de los ET. De acuerdo con estos últimos, la asignatura ha tenido buena recepción por parte de los apoderados, aun cuando en un comienzo estuvieron reticentes. 
4.1.3. Escuela rural de implementación anterior al comienzo de la asignatura (RA)

La escuela se ubica en la comuna de Padre Las Casas y entrega educación parvularia y básica en cursos combinados hasta sexto año básico. Séptimo y octavo básico tienen un solo curso por nivel. Al establecimiento asisten alumnos de dos comunidades y casi toda su matrícula es mapuche. La planta docente consta de doce profesores.

La escuela cuenta con una ET que trabajó desde las actividades del PEIB-Orígenes e integraba una dupla con las docentes de aula respectivas. Este año una docente, con especialidad en interculturalidad, ingresó al establecimiento para el cargo de profesora mentora. Como indica la directora, el objetivo buscado a través de la asignatura es que los niños aprendan mapuzugun, se encanten con la cultura y se sientan orgullosos de ella.

La directora identifica dificultades en la implementación de la asignatura en relación con tres aspectos. Primero, se encuentra el hecho de que, si bien los alumnos participan activamente en el aprendizaje del mapuzugun con la ET, no tienen la oportunidad de poner en práctica sus conocimientos, pues en la comunidad se habla poco esta lengua. En segundo lugar, identifica como dificultad la existencia de diferencias metodológicas y personales entre la ET y la nueva profesora mentora. Por último, se considera una dificultad el hecho de que la forma de trabajo de la ET no contempla ciertas prácticas exigidas por la escuela, como la planificación.

4.1.4. Escuela urbana de implementación reciente de la asignatura (UR)

Se ubica en Santiago en la comuna de San Ramón. Entrega educación parvularia y básica. Según la directora, un importante porcentaje de los alumnos posee ascendencia indígena. La escuela cuenta con 48 docentes.

La asignatura se implementó sin consulta previa a la comunidad escolar, por temor a que los padres la rechazaran o pidieran aumentar las horas de inglés. Comenzó en primero básico, utilizando una hora correspondiente a Lenguaje y Comunicación. Por parte de la directora 
y del ET, se plantea como objetivo principal de la asignatura que los niños hablen mapuzugun.

El ET asume una posición de horizontalidad con los niños en la implementación de la asignatura, priorizando la participación activa de los estudiantes por sobre la transmisión de conocimientos. Las estrategias pedagógicas declaradas por el educador son, principalmente, cantos y juegos.

Dentro de las dificultades de la asignatura, la directora y el ET mencionan el ajuste constante que ha debido realizarse sobre las planificaciones. Mencionan también, como un desafío, la necesidad de contextualizar el uso del mapuzugun al entorno urbano, así como los materiales entregados por Mineduc. Así mismo, el ET identifica como dificultad la cantidad de niños por sala y el hecho de que los contenidos de la cultura y lengua mapuche no permeen otras asignaturas y actividades de la escuela. Ambos reconocen como avance el hecho de que los niños se comuniquen en mapuzugun y lleven su uso a la familia.

\subsubsection{Escuela urbana que implementa la asignatura desde sus inicios (UI)}

Este colegio de la comuna de Osorno imparte educación parvularia, básica, básica para adultos y media (científico-humanista para jóvenes y adultos, y técnico-profesional industrial para niños). La planta docente es de 83 profesores. El director declara que en la comunidad hay una baja adscripción indígena, sin embargo, la asignatura ha tenido una buena recepción por parte de los profesores y alumnos.

Esta se implementa con cuatro horas semanales. El colegio cuenta con una profesora mentora, que también cumple la función de coordinadora intercultural, y dos ET. La clase es de carácter flexible, con conversación y cantos. El uso del mapuzugun incluye saludo, bienvenida, conversación sobre la familia, saludos de cumpleaños, entre otros.

Hay diferencias entre ET y profesora mentora con relación a los objetivos del curso. Los primeros buscan formar hablantes 
de mapuzugun, mientras que la profesora mentora promueve la formación de personas interculturales, con respeto por distintas culturas y con valoración por la propia.

El director y la profesora mentora identifican como dificultades el poco compromiso de las familias en el aprendizaje del mapuzugun, lo que ocasiona que los niños no puedan aplicar los contenidos en su vida familiar. Desde los ET, se menciona el hecho de que los contenidos no se apliquen a otras asignaturas. La profesora mentora identifica algunas dificultades provenientes de diferencias entre los ET, por ejemplo, en torno a la pronunciación, así como al hecho de que las reuniones de ET convocadas por el Mineduc sean en horario de clases. Desde la dirección se menciona los bajos sueldos asignados a los ET.

4.1.6. Escuela urbana de implementación anterior al comienzo de la asignatura (UA)

Está en la comuna de Padre Las Casas, imparte educación parvularia y básica. Se sitúa geográficamente en un sector periurbano que colinda con varias comunidades mapuche, recibiendo alumnos de todas ellas. El uso del mapuzugun no está generalizado en las comunidades. La escuela cuenta con el apoyo de la corporación indígena Choyün Folil en la realización de actividades abiertas a la comunidad, como la celebración del wiñol txipantü. La planta docente está compuesta por 22 profesores.

El colegio tiene un ET que implementa la asignatura de primero a cuarto año básico. Para alumnos de quinto a octavo básico se realizan talleres interculturales. Como indica el director y la profesora mentora, desde la escuela se considera que el objetivo principal de la asignatura es el aprendizaje y uso del mapuzugun. Desde la dirección, se identifica como una dificultad el bajo nivel de aprendizaje de mapuzugun en los estudiantes.

Son pocos los profesores que colaboran en la asignatura. En general, la recepción de los niños y apoderados es buena. Muchos de ellos participan en eventos como el nguillatun en sus propias comunidades. Pese a esto, en el pasado hubo casos de rechazo hacia la asignatura por parte de algunos apoderados. 
La implementación de la asignatura contempla la enseñanza del mapuzugun y de elementos culturales. Estos contenidos responden a dos objetivos principales que, desde las escuelas, se les atribuye a la asignatura: la revaloración y reafirmación cultural; y el aprendizaje de la lengua. Si bien, a nivel general, las escuelas combinan ambos objetivos, no se observa un énfasis en el dominio del mapuzugun por parte de los alumnos, salvo en las escuelas UR y UA, que lo plantean declarativamente.

Las escuelas que han desarrollado programas de EIB de manera previa o paralela a la implementación de la asignatura, identifican como principales dificultades la falta de retroalimentación por parte de la familia de los estudiantes para poner en práctica su aprendizaje del mapuzugun, así como problemas de carácter laboral, como el bajo sueldo de los ET e incompatibilidades de horario o conflictos al interior de la dupla pedagógica. En las escuelas donde la asignatura se implementó recientemente, se identifican como desafíos la necesidad de recontextualizar los contenidos y materiales en relación con la vida cotidiana de los educandos.

\subsection{Los Educadores Tradicionales}

A continuación, se presenta una caracterización de los ET, con base en las condiciones de su llegada a la escuela, su formación, la forma de trabajo adoptada en la dupla pedagógica y sus características en términos pedagógicos y lingüísticos. No se identificaron diferencias significativas de género en las prácticas pedagógicas ni en los contenidos impartidos por estos actores.

La mayoría de los ET participa activamente en las comunidades mapuche de las que forma parte. Por ejemplo, los educadores de las escuelas UA y RI son actualmente dirigentes en sus respectivas comunidades. Asimismo, en las escuelas UA, RR y RA, los ET fueron elegidos por la comunidad y tuvieron anteriormente una participación dirigencial en la misma.

Varios educadores mantienen una relación con sus respectivas escuelas previamente al desempeño de su cargo. Así, la educadora de la escuela RR trabajó como asistente de educación y el educador 
de UI participó en talleres interculturales realizados en el colegio. Ambos educadores de la escuela RI fueron apoderados antes de ser ET y los educadores de UI y UA fueron alumnos en las escuelas donde se desempeñan actualmente.

La mayoría de los ET fueron propuestos para el cargo por parte de algún actor del colegio, tal como el director o el sostenedor. La excepción la constituyen las escuelas UR y UI, cuyos los ET postularon al cargo de manera independiente.

Respecto de la formación de los ET, estos adquieren sus conocimientos sobre la lengua y la cultura mapuche principalmente a través de su familia y en el contacto con su comunidad. También es importante la motivación e iniciativa personal en el acercamiento a estos ámbitos. Los educadores de las escuelas RI y UR indican que estudiaron algunos libros de manera independiente. El educador de UI menciona el uso de recursos de la página web de la Corporación Nacional de Desarrollo Indígena (Conadi). Estos recursos se utilizan tanto para aprender lengua y cultura mapuche como para guiar su trabajo como ET.

Entre los casos estudiados, la forma cómo se organiza el trabajo en la dupla pedagógica es variable. En general, el esquema de trabajo contempla una planificación en conjunto, entre el profesor mentor y el ET. Así sucede, en efecto, en las escuelas RR, UI y RI. En este último caso, donde se cuenta con dos ET, la profesora mentora realiza la planificación del ET ingresado recientemente, y solo asiste al educador que posee antigüedad en su labor. Sin embargo, en la escuela RA, donde se cuenta con un horario de planificación común, ET y profesora mentora realizan sus planificaciones de manera independiente, y quien dirige el curso y las evaluaciones es la profesora mentora, con participación de la ET en determinadas actividades, correspondientes principalmente, a la conversación en mapuzugun. En estas escuelas, el horario de planificación es variable, y generalmente se sitúa después de las clases. El UI cuenta con un horario fijo para reuniones internas entre ET y profesora mentora. 
Los ET de las escuelas UR y UA planifican solos, en el primer caso, porque el educador fue contratado bajo el estatus de profesor de mapuzugun, y en el segundo, porque no existe una dupla pedagógica fija, sino por curso, de acuerdo con la disponibilidad horaria de los demás profesores.

En general, los profesores mentores asisten a los ET en los aspectos pedagógicos y en el manejo de la disciplina, trabajando en sala con ellos. En relación con lo primero, el ET propone contenidos y actividades y el profesor mentor asesora en la preparación de materiales, en la estructuración de la clase, y en las evaluaciones. En el segundo aspecto, la presencia de profesores mentores o asistentes de aula se utiliza como una forma de mantener el orden.

Las evaluaciones aplicadas a la asignatura en las escuelas estudiadas siguen metodologías convencionales: pruebas de contenido, disertaciones, guías, entre otros. Estos instrumentos se flexibilizan principalmente en torno a la oralidad: cuando los niños no obtienen buenos resultados en pruebas escritas, se practican las mismas pruebas oralmente. Salvo en el caso de RA, donde la evaluación está a cargo en su totalidad de la profesora mentora, todos los ET participan en la formulación y aplicación de las evaluaciones, ya sea trabajando solos (como en los casos de las escuelas UR y UA), o bien, colaborando con los profesores mentores.

Solamente dos educadores han cursado educación terciaria formal en el ámbito de la interculturalidad: la educadora de RR, que está estudiando la carrera de Técnico en Educación Cultural en la Universidad de Los Lagos, y uno de los educadores de RI, que asistió a un curso de competencias lingüísticas en la Universidad de La Frontera. Solo el ET de la escuela UR tiene formación en pedagogía (Pedagogía en Música). Los educadores de las escuelas RI y UA han terminado su escolaridad en programas de educación para adultos.

Salvo los educadores de UR y RR que tienen formación pedagógica, los demás han ido adquiriendo conocimientos y herramientas pedagógicas mediante el trabajo en colaboración con los profesores mentores. El ET de UA destaca su participación en el 
P900, de donde recoge experiencias que le sirven en su manejo de la asignatura.

A excepción de las escuelas donde se implementó la asignatura recientemente (RR y UR), los educadores han asistido a las capacitaciones del Mineduc. Al respecto, los educadores de las escuelas RA y UI, y uno de los educadores de RI, indican que las capacitaciones no sirven de mucho y que no son adecuadas a sus necesidades. También se afirma que son muy complejas y que falta coordinación en la difusión de la información. Pese a ello, por parte de educadores de RI y UA son valoradas como una forma de compartir experiencias entre educadores.

Respecto del dominio de la lengua que tienen los educadores, los de las escuelas donde se implementó la asignatura recientemente no son hablantes de mapuzugun. Sin embargo, ambos cuentan con una historia familiar donde se hablaba, principalmente por parte de los abuelos, lo que los dota de una familiaridad con la lengua que ha facilitado su aprendizaje. Actualmente se están formando en esta competencia. En el resto de las escuelas los educadores son hablantes de mapuzugun. La excepción la constituyen los educadores de UI, que cuentan con un nivel básico en esta lengua. En este caso, resalta que cada uno de ellos se encuentra más cercano a una variante distinta. Uno de ellos maneja más el chezugun (variante williche), mientras la otra educadora está más relacionada con el mapuzugun hablado en la Araucanía. De acuerdo con la profesora mentora, esta diferencia se traduce en algunas discrepancias entre educadores.

\subsection{Los contenidos de enseñanza impartidos en la asignatura de lengua mapuzugun}

Los contenidos de las clases de mapuzugun se asocian principalmente con prácticas culturales temáticamente agrupadas por campos semánticos, por enseñanza sobre el mapuzugun o por géneros textuales, tal como se ha identificado en la siguiente clasificación:

a. Enseñanza sobre la lengua mapuche: componentes tales como verbos, adjetivos y adverbios. Este elemento se declara principalmente en el caso RI de forma explícita. 
b. Campos semánticos: listado de palabras que pertenecen a un tema común.

- Enseñanza de valores mapuche es un campo transversal a todos los casos. En la escuela UR se enfatiza en valores interculturales.

- Elementos de la naturaleza. Este punto es tratado también en todos los casos con distintos énfasis y profundidad. Es interesante que en las escuelas urbanas UR y UI, además, se declara que enseñan en específico el uso del lawen (plantas medicinales) y que además en UR se enseñen los astros y su importancia.

- Parentesco. Se enseñan las distintas formas de nombrar a los familiares. En UA además se resalta el significado de los apellidos de la zona.

- Las partes del cuerpo (kalül). Son mencionadas como temas en RR, UA y UI, además se observaron clases que abordaron este tema en RA. En este último caso se utiliza el ülkantun memorizar ciertas partes del cuerpo.

- Números y matemática. Se observa y declara la enseñanza de los números en mapuzugun en casi todos los casos. Además, este contenido decora buena parte de las salas observadas.

- Instrumentos musicales. No solo se explica en qué consisten sino que además se utilizan en todos los casos. Es especialmente relevante el kultrung, que es usado no solo como instrumento, sino que para enseñar el meli witxan mapu en UI y RA.

- Meli witxan mapu (puntos cardinales). Este contenido también se asocia con otros aspectos de la cosmovisión mapuche y las estaciones del año. Es un tema tratado declaradamente en UI y UA, pero fue observado en otras escuelas, como RA.

- Organización comunitaria y relaciones sociales. Declarativamente se enseñan en todos los casos los roles y funciones de las autoridades tradicionales. Además, se observaron ciertas prácticas de relaciones sociales como el intercambio (txafkintu), compartir (misawün) en RA, y la forma de pedir y entregar ayuda (igkatuchen) en UI. 
- Elementos domésticos y de la ruka. En RA se enseñan las partes de la casa mapuche y cómo esta se construye; por su parte, en RI las clases se realizan regularmente en este espacio, por lo que es un recurso más que un contenido.

- Festividades, prácticas y ceremonias tradicionales. Este punto es central en los casos estudiados, pues parece ser que el wiñol txipantü ya está institucionalizado en las escuelas como parte de la celebración asociada con los pueblos originarios. En todos los casos es una práctica, pero además los estudiantes de los establecimientos rurales discuten sus contenidos y expresiones locales. Además de lo anterior, también en dichos establecimientos se tratan otras ceremonias, tales como el nguillatun o el llellipun. En este marco, conjuntamente se enseñan y practican bailes (purün), siendo, en RR y RA, objetivos de clases observadas. Otros temas que se evidenciaron en las observaciones fue en RA una clase sobre el ülkatun (canto) y sus diferentes expresiones contextuales, y en RI una clase acerca de la festividad de San Francisco.

- Otros campos semánticos. Además de lo anterior, en UR se enseña la bandera mapuche y su simbolismo; en RA conceptos como el pullü (alma) y la muerte; en RR, aspectos relativos a los sueños (pewma) y la importancia para los mapuche, entre otros puntos. Estos aspectos en ocasiones son contenidos subordinados a campos semánticos más amplios.

\section{c. Géneros textuales}

- Historias y relatos. En algunos casos, tales como en UR, UI y RI, se enseña historia mapuche y su relación con Chile. Asimismo, tanto en estas como en el resto de las escuelas, se utilizan los géneros discursivos tradicionales para narrar historias locales u obtenidas de algún libro de texto o internet. Se puede destacar que en RR se les pidió a los estudiantes que preguntaran y trajeran epew desde su casa.

- Protocolo de saludo mapuche. Se evidenció con más claridad en RA y RI, escuelas en que se les enseña a los estudiantes no solamente a saludarse entre sí, sino que además cómo entablar una conversación con autoridades tradicionales 
en distintos contextos sociales. Es posible que estos énfasis se expresen por la presencia de estas situaciones en sus contextos locales, insertos en comunidades mapuche.

En primer término, es importante señalar que los contenidos impartidos en la asignatura están referidos no tan solo a aspectos lingüísticos, relacionados con la estructura del mapuzugun y sus componentes, sino que a contenidos culturales propios del pueblo mapuche. Tal como ya se ha mencionado, los ET deben instruir a estudiantes que en su mayoría no poseen un dominio de la lengua originaria, por lo que las clases integran prioritariamente elementos básicos de la comunicación oral, como el saludo, la despedida o la manera de referirse a las personas por su condición o posición social, para luego enfocarse en la enseñanza de vocabulario situado en temas particulares al que se asocian palabras clave. Lo anterior se expresa principalmente en español en todos los casos, salvo en RA y RI en que las explicaciones se intercalan con el uso del mapuzugun.

Los elementos comunicativos básicos son presentados como rutinas pedagógicas y/o incluidas en estrategias tales como ülkantun o algún género discursivo específico. Esto tiende a repetirse en todos los casos observados, con algunas variaciones específicas. Por ejemplo, en el caso de RA, el saludo se realiza mirando en media luna hacia el este (salida del sol) y un representante saluda uno por uno a los integrantes del curso; en RI, es el logko del curso quien encabeza el saludo y abre la clase. En el resto de los casos, no se le da mayor énfasis a este punto e incluso estas rutinas se circunscriben solamente al espacio de la asignatura o a alguna festividad, como el wiñol txipantu, no así en los casos mencionados donde el uso de estos protocolos se utiliza incluso en otras instancias de la escuela.

Se evidenció en los casos observados que los educadores tomaron como una decisión pedagógica que el conocimiento mapuche (kimün) llevado a la escuela —que a diferencia del pensamiento moderno occidental no está segmentado en disciplinas ni compartimentado en áreas- debió establecer límites temáticos para poder materializarse en clases. Sin embargo, muchas veces estos campos semánticos se traspasan de un lado para otro y se 
complementan. Por este motivo, es recurrente que en las clases algunos campos semánticos se traslapan o se deben mencionar para explicar algunos aspectos. Un ejemplo claro es que para explicar una festividad se debe hacer mención a la organización comunitaria mapuche y a elementos comunicativos. En este sentido, el abordaje de diferentes temáticas en la enseñanza del mapuzugun viene a fragmentar dicha perspectiva de desvinculación, entendiendo que en la cultura mapuche el conocimiento no está segmentado.

Respecto de la selección de los contenidos, el contexto resulta relevante, sobre todo, en aquellos de carácter histórico. Se reflexiona en torno a una enseñanza situada, local en todos los casos, enfatizando en la relevancia del entorno. De igual modo, la historia de Chile se construye implicando la historia del pueblo mapuche, de tal modo de no relatar solo una perspectiva de realidad. En efecto, la historia del mundo mapuche no está escindida de la enseñanza de la lengua, entendiendo que el estado de minorización que caracteriza a las lenguas indígenas se construyó al alero de procesos sociohistóricos.

Asimismo, la responsabilidad de la selección de los contenidos recae en el educador o el docente mentor, dependiendo del tipo de relación que se construye al interior de la dupla. Así, en las escuelas rurales es el PM quien determina el contenido a tratar en cada una de las clases; en la siguiente cita se advierte la dificultad que el ET tiene respecto de la dosificación de contenidos, por ejemplo:

(...) le da mucha vuelta a una cosa, a un contenido. Por ejemplo, el epew, lo estuvimos pasando ¿cuánto tiempo? Y pasar tres epew al tiro... que los niños, con uno que les pasen, y desglosar, eso a mí me parecería bien, pero no pasarles tres epew de un viaje. Yo creo que ahí, un poco se confunden (RA).

En tanto, el resto de los establecimientos son los ET quienes seleccionan los contenidos y los adecuan al contexto. Existe una tensión respecto del foco de la asignatura. La dupla pedagógica reconoce que el programa busca que el niño hable en lengua indígena, sin embargo, la enseñanza se direcciona, principalmente, hacia aspectos valóricos y al conocimiento de la cultura en materia de contenidos. 
En relación con los programas de estudio de la asignatura Lengua Indígena, el uso de estos es disímil en lo concerniente a los contenidos abordados en las clases. Si bien en la escuela RI los conocen, no son utilizados, puesto que tanto educador como profesora mentora sostienen que es el contexto el que determina qué contenidos abordar. En tanto, en el resto de los casos analizados, se toman como referencia algunos, que luego son contextualizados de acuerdo con las características del entorno. Esta particularidad es más explícita en las escuelas urbanas, donde los educadores reconocen que los programas están descontextualizados, puesto que refieren principalmente al mundo rural.

(...) me dijeron "esto es lo que tú tienes que enseñar", y yo leí el programa, reconozco que lo tomé en cuenta, o sea, el año escolar lo divido en todas las unidades que ellos plantean, y luego las desarrollo, pero las desarrollo desde mi mirada, desde las cosas que yo sé, y de las cosas que son... que tienen sentido para mi parecer, bajo mi criterio, desarrollarlas en clases porque el programa, según mi opinión está súper descontextualizado, porque está pensado para una, para el mundo rural (UR).

Por último, cabe destacar que la enseñanza de los contenidos no está escindida de los aspectos culturales, principalmente, en lo concerniente a la naturaleza.

\subsection{Reflexiones sobre el aprendizaje del mapuzugun}

Cobra especial relevancia el ejercicio de reflexión lingüística llevada a cabo por la dupla pedagógica, sobre todo, en los establecimientos donde circula la variante dialectal chezugun (RR y UI). A la hora de enseñar lengua, el criterio es marginar palabras que han sido castellanizadas, aun habiendo sido refonemizadas.

Aquí en este sector se dice muchulla, pero si ustedes van a un sector más al norte se dice reñma. Así hacemos la variante, hay palabras que, en Temuco se dice, no sé, bailar se dirá purrun, pero acá se dice bailañe. Entonces, yo no voy a tomar bailañe, que es una palabra castellanizada (RR). 
Teníamos el problema con chaino, por ejemplo, porque chaino suena como chao. Entonces yo le digo, ¿será chaino, o será sraino? Porque yo he escuchado sraino, en la costa la "r" se arrastra. Cuando cantamos suena sraino, entonces también hay que buscar el origen de la palabra que con el tiempo se ha ido castellanizando, hay que tener cuidado. Eso nos cuesta a nosotros, cuesta bastante (RR).

No solo se insiste en la recuperación de la fonética que caracteriza la variedad dialectal de la zona, sino que también quienes enseñan la lengua indígena reconocen que este proceso es complejo en un contexto sociolingüístico donde la vitalidad del chezugun es baja.

A lo anterior, se suma la escasa circulación de material educativo en chezugun. Aunque la tendencia es utilizar material pedagógico en mapuzugun, se explicitan los aspectos propios de la variante mencionada a fin de que los estudiantes comprendan la coexistencia de variedades dialectales.

Uno termina haciendo mapuzugun porque está disponible en todas partes, en los libros, en los textos (...) Un día una reflexión a la educadora: ¿Qué posibilidades tiene un niño de aquí de hablar con un hablante de chezugun? ¿Y con uno de mapuzugun? 'Tiene razón', me dijo. Las posibilidades que tienen ellos, es con uno de mapuzugun, porque uno de chezugun pueden ser dos o tres los que van a venir a la sala, de mapuzugun van a encontrar muchos. Entonces nosotros les enseñamos las dos variantes (UI).

Por otra parte, entre los contenidos declarados por los educadores, se releva el uso de relatos locales y cuando estos provienen de otras regiones, como en el caso de los programas de estudio, son adecuados al contexto, de tal modo de dotarlos de significado. Esta tendencia está presente en las diferentes escuelas, aunque con mayor énfasis en los establecimientos RR y UI, por tratarse de una zona williche y en UR, por su carácter urbano.

(...) todas las historias que vienen son de Panguipulli, son de Boroa, de Tirua, pero no está, hasta el momento no lo he visto, 
no está el tratado de las canoas, no están las misiones cuando se instalaron, esos son temas que acá sí se trabajan, entonces eso nosotros lo trabajamos de forma personal (UR).

\subsection{Las estrategias de enseñanza desarrolladas en la asignatura de lengua mapuzugun}

Sobre la base de la codificación de las entrevistas se han clasificado las estrategias de enseñanza en dos tipos: las vinculadas con la cultura mapuche y aquellas no vinculadas directamente con esta cultura.

Las estrategias de enseñanza mapuche representan metodologías que provienen de prácticas culturales que se han realizado tradicionalmente en las comunidades mapuche. Con la implementación de la asignatura en las escuelas, las prácticas culturales propias de las comunidades se han adaptado para responder a un contexto nuevo y ajeno, la escuela chilena: por tanto, la consideración de una determinada estrategia como estrategia mapuche viene dada por su vinculación con prácticas culturales propias $^{10}$, con independencia de la existencia de cierto grado de cambio provocado por la adaptación al sistema escolar.

Las estrategias de enseñanza no vinculadas a la cultura mapuche son acciones intencionadas funcionales al logro de los aprendizajes y que se encuentran diseñadas para instrucción en el aula, sin tener un correlato con prácticas culturales propias de las comunidades mapuche.

Considerando lo anterior, dentro de las estrategias vinculadas con la cultura mapuche, hemos identificado las siguientes:

a. Actividades manuales: por ejemplo, trabajos en greda (metawe) o la construcción de la ruka (casa). Estas actividades aparecen en UA y RA.

10 Las prácticas desarrolladas en la escuela occidental son también reflejo de la vinculación de una comunidad particular con la escuela. En este sentido, las prácticas identificadas como mapuche lo son efectivamente, al igual que los bailes folclóricos en la educación básica involucran una vinculación de una manifestación cultural particular. Por ejemplo, la enseñanza de la cueca se manifiesta de diferentes maneras en contextos diversos. 
b. Purün: por ejemplo, ensayo y muestra en público de bailes como el choyke purün. Esto se desarrolla en dos de las escuelas urbanas (UA, UI).

c. Conversación con autoridades tradicionales: invitación a la escuela o visita a autoridades tradicionales como el logko o la machi. Esta práctica aparece en las escuelas rurales y urbanas con desarrollo anterior a la asignatura.

d. Conversación: los ET de las escuelas rurales con implementación desde el año 2010 y anterior de la asignatura relevan el uso de la conversación en general como una estrategia pedagógica.

e. Uso de géneros tradicionales: interacción mediada por el uso de géneros propios de la cultura mapuche, en donde destacan el ngülam (consejo), el nütxam (conversación), el konew (adivinanza), el piam (relato sobre algún suceso). Los más utilizados son el epew (cuentos tradicionales) y el ülkantun (canto). El uso de estos aparece en todas las escuelas.

f. Salidas pedagógicas: visitas a lugares de importancia para la cultura mapuche como nguillatun, palin, lugares donde sacar materiales para hacer rukan y ferias indígenas.

g. Uso de vestimenta mapuche: los ET llevan vestimenta tradicional mapuche o reproducciones de esta y se visten para ocasiones especiales o para aprender los nombres de la ropa y las joyas. El uso de vestimenta aparece en las escuelas urbanas con implementación anterior desde el 2010.

Por su parte, dentro de las estrategias no vinculadas directamente con la cultura mapuche, se han identificado las siguientes:

a. Uso de guías de enseñanza: los ET de la escuela UI y la escuela RA declaran utilizar estas estrategias.

b. Uso de libro de textos: esta estrategia es mencionada en todas las escuelas urbanas.

c. Medios audiovisuales: las escuelas urbanas con implementación inicial y con implementación anterior al año 2010 y la escuela rural anterior declaran utilizar medios audiovisuales. 
d. Uso de dibujos traídos por el educador: las escuelas urbanas con implementación desde el año 2010 y con implementación anterior utilizan los dibujos como estrategia pedagógica.

e. Teatro o dramatización (representaciones, diálogos, etc.): la escuela urbana con implementación reciente, y las escuelas rurales con implementación desde el 2010 y anterior declarar utilizar esta estrategia.

Las estrategias descritas son las declaradas por los educadores cuando mencionan aquellas que utilizan en aula, sin embargo, la observación permitió constatar que en varios casos los ET o profesores mentores utilizan estrategias que no declaran en sus entrevistas. Por ejemplo, la conversación solo fue relevada en escuelas rurales, pero también pudimos observar escuelas urbanas en las que el ET utiliza la conversación con los estudiantes como una estrategia pedagógica para transmitir contenidos en la clase. Por ejemplo el ET de la escuela con implementación reciente de la asignatura utiliza la conversación con los estudiantes para que entiendan la importancia del wiñol txipantü. El ET les pregunta a los estudiantes por sus experiencias con el invierno y permite una interacción que no es habitual dentro de las interacciones de aula en la educación primaria ${ }^{11}$. En este sentido, se pudo relevar el uso de la conversación como una estrategia que puede relacionarse con el tipo de interacciones culturales propias de las comunidades.

Respecto de los usos que se le dan a cada estrategia, la observación de clases permitió entender los fines con los que las diferentes estrategias son llevadas al aula. El dibujo, por ejemplo, se utiliza como evidencia de aprendizajes en todas las escuelas urbanas.

Con respecto a las estrategias mapuche, vale la pena destacar que en todas las escuelas se utiliza al menos una estrategia mapuche de enseñanza. Dentro de estas, el uso de géneros tradicionales es la

11 La literatura ha descrito que típicamente la interacción de aula entre profesor y estudiante se desarrolla a partir del esquema: Pregunta - Respuesta - Retroalimentación. Esta secuencia ha sido denominada como IRF por sus siglas en inglés (Cazden, 1988; Sinclair \& Coulthard, 1975). 
más utilizada (en todos los colegios con excepción de UA). Resulta interesante destacar, en primer lugar, que las escuelas que presentan mayor diversidad de estrategias mapuche (cinco de las siete) son la escuela UA y la de RA. Por otro lado, aquellas que registran menos diversidad (una de siete) son las escuelas RR y UR. En este sentido, pareciera ser que el lugar geográfico no se correlaciona con la diversidad de estrategias mapuche utilizada. Ahora bien, es importante destacar que un factor que puede tener algún grado de influencia en la posibilidad de realizar estrategias mapuche tiene que ver con la ubicación de la escuela en el territorio ancestral mapuche, donde más allá de su ubicación rural o urbana, pueden gestionarse con mayor facilidad actividades manuales con productos como el junquillo, conversaciones con autoridades tradicionales y salidas pedagógicas a lugares de relevancia. Así, por ejemplo, obsérvese lo señalado por el profesor mentor de la escuelas UI, espacio donde se releva la posibilidad de la escuela de asistir a sectores rurales tradicionales y donde pueden vivir las prácticas mapuche en terreno: "Se va al nguillatun de Pualhue en San Juan De La Costa, esta escuela va a ese nguillatun".

Obsérvese el contraste con lo señalado por el educador de UR, escuela urbana de Santiago, donde se destacan los esfuerzos que tiene que hacer para poder contextualizar la enseñanza de la lengua en esta realidad:

Por ejemplo si tengo enseñar plantas medicinales, cómo puedo enseñar de plantas medicinales si no estoy en el campo, entonces tengo que... ingeniármelas de alguna manera, tratar de usar lo que ellos saben o sea por ejemplo, ya niños ustedes, nómbrenme... remedios... cómo puedo decirlo, o les pregunto así, alguna vez cuando ustedes se han enfermado de la guata por ejemplo, qué han tomado, qué les ha dado su mamá, entonces los niños empiezan a decir más o menos los remedios que conocen, pero por eso cuesta mucho contextualizarlo acá en el mundo urbano porque la cultura mapuche igual está como decirlo, o se entiende siempre en la ruralidad. 
De todas maneras, más allá de la presencia en territorio tradicional mapuche, el factor que parece ser decisivo en la diversidad de las estrategias utilizadas es la antigüedad de la enseñanza de la lengua mapuche. Las dos escuelas que cuentan con un mayor grado de diversidad implementan la enseñanza de la lengua mapuche desde antes de 2010 y las dos escuelas que cuentan con un menor grado de diversidad son las que cuentan con la asignatura desde épocas recientes. Justamente, en un punto intermedio se encuentran las que la implementan desde el año 2010.

En relación con las estrategias no mapuche, se registra ligeramente una mayor diversidad en las escuelas urbanas, que presentan tres de cinco, que en las rurales, puesto que si bien RA tiene también tres de las cinco estrategias registradas, tanto RI como RR registran solamente una actividad — coincidentemente, en ambas solo se usa la dramatización. Por otro lado, no se registra una correlación entre antigüedad de la implementación y diversidad de estrategias en este punto. Es posible que un factor decisivo para este punto tenga que ver con las condiciones de implementación de la escuela en cuestión, sobre todo con la posibilidad de contar con recursos audiovisuales, por ejemplo, y también con la actitud del educador con respecto a la incorporación de tecnologías a la enseñanza del mapuzugun.

Respecto de los recursos educativos que se utilizan en la asignatura, destaca la diversidad de materiales con que trabajan. ET o profesores mentores destacan el uso de materiales concretos como elementos que los estudiantes deben traer desde sus hogares (RA) o elementos de la naturaleza (UR, RR). Por ejemplo, en la escuela rural con iniciativas anteriores (RA), observamos cómo el profesor mentor explicó los conceptos de misawün y txafkintu a través de un intercambio real de artículos. Por otro lado, también destaca el uso de recursos audiovisuales lo que fue relevado por todos los ET o profesores mentores. Este recurso resulta relevante puesto que los ET lo destacan como uno que los estudiantes valoran (ET RI, UR) y que les permite compartir con los estudiantes el material que encuentran en Youtube (ET, UR), o en internet (PM, UA). Además del material didáctico que los ET o PM rastrean en internet, resulta relevante destacar que en todos los casos los ET y PM crean o adaptan 
materiales educativos con el fin de tener una mayor cantidad de recursos para trabajar los contenidos en el aula. La construcción de materiales propios adquiere relevancia en tanto este se utiliza primordialmente para incorporar contenidos culturales. Por ejemplo, los ET declaran crear poemas (ET, UR), guías (ET RI, UA, UI) o epew (ET RI, UI, RA). Otro de los recursos educativos que destacan como relevantes, en tanto que aparecen en cuatro de los casos observados (UI, UR, UA, RI), es el uso de instrumentos musicales mapuche. Por lo general, los docentes utilizan sus propios instrumentos, los que llevan al aula para explicar parte de las tradiciones mapuche o para acompañar las canciones que enseñan a los estudiantes. Por último, el uso de los textos escolares se releva en cuatro de los seis casos observados (UI, UA, UR, RR).

El análisis de las entrevistas y observaciones de clase permitió identificar al menos dos tipos de estrategias de enseñanza: las estrategias vinculadas con la cultura mapuche y aquellas no vinculadas directamente con esta cultura. En este punto cabe destacar que los ET manifiestan esta distinción al identificar aquellas estrategias propias del concepto de educación occidental:

Yo creo que eso es una ventaja de la clase, hay ventajas también del concepto de educación occidental, eso del inicio, dar un inicio, un desarrollo y un cierre [...] esa estructura la mantengo bastante, yo creo que hace que el conocimiento sea ordenado... si ya planteamos el mapuzugun como un idioma, también hay que darle esa pequeña rigurosidad (ET, UR).

En este sentido, el ET identifica una clara diferencia entre las estrategias de la cultura occidental y la cultura mapuche.

\section{Discusión y conclusiones}

Los casos estudiados constituyen distintos contextos en los que se desenvuelve el trabajo de los ET, configurados por las características de la comunidad, por el momento en que se encuentra la escuela con relación a la implementación de la asignatura y por cómo esta se concibe, entre otros. Asimismo, los educadores poseen distintas 
trayectorias laborales y formativas y diferentes grados de cercanía con la lengua y la cultura mapuche.

En la mayor parte de los casos estudiados, los ET poseen un alto grado de vinculación con la comunidad en la que trabajan y un conocimiento de la lengua y la cultura mapuche adquirido a través de la relación con su familia y su comunidad. Este conocimiento les permite trabajar aspectos como conversaciones protocolares mapuche y las ceremonias, a partir de cantos, juegos y otras estrategias. Los educadores reciben asesoría de los profesores mentores en aspectos pedagógicos, mediante formas de trabajo más o menos colaborativas, aunque también se registran casos en que trabajan solos.

No obstante sus diferencias en el dominio de la lengua y la cultura, así como en el manejo de los aspectos pedagógicos, los ET coinciden en ver la asignatura como una forma no solo de enseñar la lengua, sino también de revalorizar la cultura mapuche.

En relación con lo anterior, resulta interesante destacar la primacía que tiene la enseñanza de contenidos culturales sobre los lingüísticos, lo que resulta contradictorio respecto de los objetivos propios de la asignatura Lengua Indígena. Como proyección de la presente investigación, planteamos la necesidad de profundizar si esta primacía se vincula solamente con las preferencias de los ET, o si también influyen en ella aspectos como la falta de iniciativas de capacitación de enseñanza de la lengua mapuche como segunda lengua o la necesidad de aumentar la colaboración entre la dupla pedagógica en términos metodológicos.

Sobre los contenidos de enseñanza, se observa que hay gran similitud entre los casos, tanto urbanos como rurales. Los educadores seleccionan principalmente contenidos culturales, incluso tomando como referencia en algunos casos los programas de estudio, aunque son adaptados a la realidad local, salvo el caso de RA donde es una profesora mentora la que toma las decisiones curriculares. En este sentido, los educadores hacen uso de la autonomía que presentan para implementar la asignatura tomando decisiones de acuerdo con el conocimiento adquirido en su historia de vida. 
Es llamativo, además, que la selección de contenidos cobre gran relevancia en las decisiones pedagógicas de los educadores pues, debido al bajo dominio de base de los estudiantes que atienden, la enseñanza de elementos propiamente lingüísticos pasa a un segundo plano en términos conceptuales, compensándose esta situación con un enfoque comunicativo de las clases y énfasis en las competencias básicas de la oralidad.

Respecto de las estrategias de enseñanza, los resultados evidencian la amplia variedad de estrategias y recursos con que la dupla pedagógica implementa la asignatura. Sin embargo, esta variedad puede ser analizada desde su vinculación con la cultura mapuche. En la implementación de la asignatura, la enseñanza de la lengua mapuche se transforma en un espacio de tensión en donde interactúan dos concepciones diferentes de educación que son adaptadas en forma activa y creativa por el educador en un contexto adverso: es en este punto, donde más se revela la necesidad de planes y programas contextualizados y de procesos de formación profesional, tanto para educadores como profesores mentores, que permitan apoyar y sistematizar este proceso de adaptación/tensión. Por una parte, los educadores incorporan metodologías y estrategias de enseñanza provenientes de la cultura de la escuela, adaptándolas y haciéndolas funcionales para la enseñanza de la lengua mapuche, pero también son agentes que promueven la incorporación de prácticas culturales mapuche a la escuela que, al igual que las estrategias no mapuche, son redireccionadas hacia el objetivo de la enseñanza de la lengua y la cultura en un contexto nuevo. En otras palabras, la tensión se produce porque en este proceso creativo, que demanda una serie de competencias por parte de los educadores, muchas veces no se observa un respaldo institucional que apoye, fomente y sistematice las diferentes experiencias.

Por otro lado, la estrategia vinculada con la cultura mapuche es una actividad propia y específica de la vida de las comunidades mapuche y aplicada, pero no diseñada especialmente para la enseñanza de la lengua en la escuela. Es relevante destacar que las prácticas culturales mapuche son llevadas a la sala como una actualización que fomenta la revitalización de los géneros y estrategias que relevan 
la conceptualización mapuche de educación. La utilización de los géneros discursivos es un ejemplo de cómo las prácticas culturales como la conversación o el epew son llevadas al aula con diversos fines. En este sentido, las estrategias vinculadas con la cultura mapuche se definen como aquellas estrategias de enseñanza que provienen de una práctica cultural comunitaria originada fuera del aula. Se trata de prácticas culturales que se utilizan en el aula con el fin de que los estudiantes aprendan a realizar actividades culturales propias de la cultura mapuche.

\section{Referencias}

Bryman, A. (2004). Social research methods. Oxford, NY: Oxford University Press.

Carvajal, R. y Lafferte, M. (2014). Presencia indigena en el sistema escolar chileno: caracterización estadística. Texto borrador. Santiago.

Casarini, M. (1999). Teoría y diseño curricular. México D.F.: Editorial Trillas.

Castillo, S. y Loncon, E. (2015). Noción de Educadores Tradicionales mapuche en contextos urbanos, desde la perspectiva de la dupla pedagógica. Contextos, 33, 29-46.

Cazden, C. (1988). Classroom discourse. The language of teaching and learning. Portsmouth, NH: Heinemann.

Centro de Políticas Comparadas de Educación, CPCE. (2012). Educación para preservar nuestra diversidad cultural: desafios de implementación del Sector de Lengua Indigena en Chile. Santiago de Chile: CPCE, Unicef y Mineduc.

Cortés, P. y Concha, R. (2013). Proyectos interculturales en establecimientos urbanos: Región Metropolitana y Biobío. Estudio exploratorio (Informe final preparado para el Ministerio de Educación y Unicef). Santiago.

Creswell, J. (2009). Research design: Qualitative, quantitative, and mixed methods approaches. Los Angeles, London, New Dheli, Singapore, Washington D.C.: SAGE Publications.

Dirección de Presupuestos, Dipres. (2013). Programa aplicación de diseño curricular y pedagógico intercultural bilingüe. Recuperado de http://www.dipres.gob.cl/595/w3-propertyvalue-22041.html

Espinoza, M. (2016). Contextos, metodologías y duplas pedagógicas en el Programa de Educación Intercultural Bilingüe en Chile: una evaluación 
crítica del estado del debate. Pensamiento Educativo, Revista de Investigación Educacional Latinoamericana, 53(1), 1-16. https://doi.org/10.7764/pel.53.1.2016.11

Friese, S. (2012). Qualitative Data Analysis with ATLAS.ti. Los Angeles, London, New Dheli, Singapore, Washington D.C: SAGE Publications.

Gundermann, H., Canihuan, J., Clavería, A., y Faúndez, C. (2008). Perfil sociolingüístico de comunidades mapuches de la Región del Biobio, Araucanía, Los Ríos y Los Lagos. Santiago de Chile: Conadi-UTEM.

Gundermann, H., Canihuan, J., Clavería, A., y Faúndez, C. (2009). Permanencia y desplazamiento, hipótesis acerca de la vitalidad del mapuzugun. Revista de Lingüística Teórica y Aplicada 47(1), 37-60. https://doi.org/10.4067/s0718-48832009000100003

Gundermann, H., Canihuan, J., Clavería, A., y Faúndez, C. (2011). El mapuzungun, una lengua en retroceso. Atenea, 513, 111-131. https://doi.org/10.4067/s0718-04622011000100006

Ibáñez, N., Rodríguez, M., y Cisternas, T. (2013). La Educación Intercultural desde la perspectiva de docentes, educadores tradicionales y apoderados mapuche de la Región de la Araucanía: Una co-construcción (Informe final). Santiago de Chile: Fonide. Recuperado de

https://centroestudios.mineduc.cl/wp-content/uploads/ sites/100/2017/07/F811345-Informe-Final-Iba\%C3\%Blez-UMCE.pdf

Lara, M. (2012). El mapuzugun y su enseñanza. Sembrando Ideas, 6, 8-19.

Lave, J. (2009). The practice of learning. In Contemporary Theories of Learning. 200-208. New York: Routledge

Lofland, J. \& Lofland, L. (1995). Analyzing social setting: A guide to qualitative observation and analysis. London: Wadsworth.

Luna, L., Benavides, P., Gutiérrez, P., Alchao, M., y Dittborn, A. (2014). Aprender lengua y cultura mapuche en la escuela: estudio de caso de la implementación del nuevo Sector de Aprendizaje Lengua Indígena desde un análisis de recursos educativos. Estudios Pedagógicos, 40(2), 221-240. https://doi.org/10.4067/s0718-07052014000300014

Organización de las Naciones Unidas, ONU. (1948). Declaración Universal de Derechos Humanos. Recuperado de http://www.ohchr.org/EN/UDHR/Documents/UDHR_Translations/ spn.pdf

Organización de las Naciones Unidas, ONU. (1966). Pacto Internacional de Derechos Civiles y Políticos. Recuperado de http://www.ohchr.org/SP/ProfessionalInterest/Pages/CCPR.aspx 
Organización de Naciones Unidas, ONU. (1989). Convención sobre los derechos del niño. Recuperado de

ht t ps://www. unicef. org/argentina/spanish/7. Convencionsobrelosderechos.pdf

Organización de las Naciones Unidas, ONU. (2007). Declaración de las Naciones Unidas sobre los derechos de los pueblos indígenas. Recuperado de http://www.un.org/esa/socdev/unpfii/documents/DRIPS_es.pdf

Organización de las Naciones Unidades para la Educación, Ciencia y Cultura, Unesco. (2000). Informe sobre la educación en el mundo. Recuperado de http://es.unesco.org/gem-report/

Organización de las Naciones Unidades para la Educación, Ciencia y Cultura, Unesco. (2005). Declaración universal sobre bioética y Derechos Humanos. Recuperado de http://portal.unesco.org/es/ev.php-URL_ID=31058\&URL_DO=DO_ TOPIC\&URL_SECTION=201.html

Organización Internacional del Trabajo, OIT. (1989). Convenio sobre pueblos indígenas y tribales, 1989 (núm. 169). Recuperado de http://www.ilo.org/dyn/normlex/es/f?p=NORMLEXPUB:12100:0::NO ::P12100_ILO_CODE:C169

Quidel, G. (2011). Estrategias de enseñanza de la lengua mapuzugun en el marco del PEIB Mineduc-Orígenes (Comuna de Padre de Las Casas, IX Región-Chile). Cuadernos Interculturales, 9(16), 61-80.

Quilaqueo D. y Quintriqueo, S. (2010). Saberes educativos mapuches: un análisis desde la perspectiva de los kimches. Polis, 21, 1-21. https://doi.org/10.4067/s0718-65682010000200016

Quilaqueo, D. y Torres, H. (2013). Multiculturalidad e interculturalidad: desafíos epistemológicos de la escolarización desarrollada en contextos indígenas. Alpha, 37, 285-300.

https://doi.org/10.4067/s0718-22012013000200020

Robson, C. (1993). Real world research: A resource for social scientists and practitioner researchers. Cambridge: Blakewell.

Sinclair, J. M. \& Coulthard, M. (1975). Towards an analysis of discourse: The English used by teachers and pupils. Oxford: Oxford University Press.

Solé, I. (1994). Estrategias de lectura. Barcelona: Graó.

Sotomayor, C., Allende, C., Castillo, S., Fuenzalida, D., y Hasler, F. (2013). Competencias y percepciones de los ET mapuche en la implementación del Sector de Lengua Indígena Mapuzugun (Informe final). Santiago de Chile: Fonide. Recuperado de http://sgdce.mineduc.cl/descargar.php?id_doc=201604111614500 
80 ESTUDIO DE CASOS DE LA ENSEÑANZA DE LA LENGUA Y CULTURA MAPUCHE Y SU IMPLEMENTACIÓN COMO ASIGNATURA DEL CURRÍCULO DE EDUCACIÓN BÁSICA - M. Calderón, S. Castillo, D. Fuenzalida, F. Hasler, H. Mariano y C. Vargas

Sotomayor, C., Allende, C., Castillo, S., Fuenzalida, D., y Hasler, F. (2014). Competencias y percepciones de los ET mapuche en la implementación del Sector de Lengua Indígena Mapuzugun. Recuperado de www.ciae.uchile.cl/download.php?file=doctrabajo/17-012015.pdf

Recibido: 01/09/2017

Aceptado: 10/11/2017 